were tested for MG using the Fast-track Diagnostics ${ }^{\mathrm{TM}}$ urethritis PCR.

Results 461 patients were tested for MG. 30/461 (6.5\%) were positive. Median age was 30 years(range 16-53) and more MG-positive males (26/30) than females (4/30) were identified. 1/4 females provided a cervical sample and 3/4 vaginal swabs. Of males, $1 / 26$ provided a penile swab, 3/26 rectal swabs, and 22/26 (84.6\%) gave urine samples. All females self-identified as heterosexual. 10/26 (38\%) men self-identified as men who have sex with men (MSM); 6/30 (20\%) patients were known to be HIV-positive, all of whom were male and 5/6 (83\%) were MSM. 9/30 (30\%) patients were treated with $1 \mathrm{~g}$ single dose azithromycin and $5 / 30(16.7 \%)$ received a regimen of azithromycin $500 \mathrm{mg}$ stat followed by $250 \mathrm{mg}$ od for 4 days. Tests of cure were done in 13/30 (43.3\%). 4/13 (30.7\%) remained positive and all received moxifloxacin, which was curative.

Conclusion We found $\mathrm{MG}$ in symptomatic patients attending our service. Many patients were treated with single dose azithromycin which may be insufficient to clear infection and lead to acquired resistance. Local protocols for persistent urethritis and PID should include routine testing for MG, and newer and better access to diagnostics are urgently needed to support this.

\section{P013 WHEN IS A HERNIA NOT A HERNIA AND LYMPHOMA NOT LYMPHOMA?}

Matthew Hamill. Berkshire Healthcare NHS Foundation Trust, Slough, UK

\subsection{6/sextrans-2016-052718.68}

Background/introduction Lymphogranuloma vereneum (LGV) is a relatively common cause of proctitis and other gastrointestinal symptoms in men who have sex with men (MSM). Other symptoms and signs may present and unless a careful sexual history is taken STI may not be considered in the differential diagnosis.

Aim(s)/objectives To illustrate the potential for mis/inaccurate diagnosis of groin swellings in sexually active MSM and provide a case that can be used for teaching primary care, surgical, oncology and histopathology colleagues.

Methods We present a case of a 55 year old HIV-infected MSM who presented to surgical colleagues with left groin swelling.

Results The patient underwent open surgery to repair an inguinal hernia. At surgery he was found to have significant inguinal lymphadenopathy. Histopathological analysis at the regional pathology centre identified a B cell lymphoma and referral was made to a haematologist to start anti-cancer therapy. In the interim the patient attended our GUM service, was diagnosed with rectal LGV and treated with antibiotics. His lymphadenopathy resolved and staging CT was negative.

Discussion/conclusion Careful consideration of the differential diagnosis of inguinal swelling should be undertaken and STI excluded prior to general anaesthesia and operative procedures whenever possible. Had this patient not attended his GUM clinic he may have undergone potentially toxic chemotherapy to treat LGV infection. This case serves to illustrate the need for open communication between GUM and other medical colleagues.

\section{P014 AN AUDIT OF PREVENTION OF MOTHER TO CHILD TRANSMISSION SERVICES WITHIN A ANTENATAL CARE FACILITY IN A RURAL HEALTH CLINIC IN SWAZILAND}

Sarah Blacker. University of Birmingham Medical School, Birmingham, UK

\subsection{6/sextrans-2016-052718.69}

Background/introduction Swaziland is recorded to have the world's highest HIV prevalence amongst adults and pregnant women. To address this epidemic Swaziland's Ministry of Health $(\mathrm{MOH})$ has adopted the WHO four pronged approach to reducing new HIV infections in women and children.

Aim(s)/objectives To audit whether prevention of mother to child transmission (PMTCT) services at a rural health clinic in Swaziland meets the $2010 \mathrm{MOH}$ targets.

Methods Retrospective data was collected for all women accessing ANC services at the clinic from 1st Feb to 25th May 2015 analysis was performed using Microsoft Excel 2013

Results 29 women accessed ANC services in this time period, $11(37.9 \%)$ were known HIV positive and a further $4(22.2 \%)$ tested positive at presentation. The clinic achieved a HIV testing rate of $94.4 \%$ (target $100 \%$ ) and a partner testing rate of $11.1 \%$ (target 50\%). 93.3\% (15) of HIV positive women received efficacious antiretroviral therapy (target 97\%) and 93.3\% (15) of exposed infants were initiated on appropriate prophylaxis (target 95\%).

Discussion/conclusion This audit has identified areas where action is required for ANC services at the clinic to meet $\mathrm{MOH}$ targets. Early HIV diagnosis and partner testing must be prioritised to reduce new born infections. Access to necessary treatment should be improved by establishing links to antiretroviral clinics.

\section{P015 BASHH MSM SIG CLINIC SURVEY; TESTING AND VACCINATION}

${ }^{1}$ Dan Clutterbuck*, ${ }^{2} \mathrm{MSM}$ Special Interest Group. ${ }^{1}$ Chalmers Centre, Edinburgh, UK; ${ }^{2}$ BASHH, London, UK

\subsection{6/sextrans-2016-052718.70}

Background/introduction/Aim(s)/objectives Our aim was to investigate practice across the UK in aspects of the clinical care of MSM who are HIV negative or of unknown status where evidence is absent, or guidance varies.

Methods An online questionnaire was drafted by the MSM SIG, tested by BASHH CGC members, revised and distributed to BASHH, FSRH members and CSP audit sites for one month to $31^{\text {st }}$ October 2015.

Results There were 149 complete responses. Only 40\% of respondents had a written protocol or policy on recall for HIV/ STI testing of which $23 \%$ had an automated system to recall patients for testing. 50\% routinely test for HIV at syphilis follow up. 90\% of respondents report using both NAAT and culture for GC in contacts of gonorrhoea and $20 \%$ use both in asymptomatic men. $33 \%$ test anatomical sites according to sexual contact history. Self-taken throat (rectal) swabs for GC/Ct NAAT were used never by $26 \%$ (3\%) and routinely in $18 \%(22.5 \%)$. $100 \%$ routinely test MSM for Hepatitis B exposure and over $50 \%$ for Hepatitis C. $78 \%$ routinely check HepB sAb levels 